\title{
Torques and the Related Meridional and Vertical Fluxes of Axial Angular Momentum
}

\author{
JOSEPH EGGER \\ Meteorologisches Institut, Universität München, Munich, Germany \\ KLAus-Peter Hoinka \\ Institut für Physik der Atmosphäre, DLR, Oberpfaffenhofen, Germany
}

(Manuscript received 19 April 2004, in final form 21 July 2004)

\begin{abstract}
The budget equation of the zonally averaged angular momentum is analyzed by introducing belts of $1000-\mathrm{km}$ width to cover the meridional plane from pole to pole up to an altitude of $28 \mathrm{~km}$. Using ECMWF Re-Analysis (ERA) data the fluxes of angular momentum are evaluated as well as the mountain and friction torques per belt. Generalized streamfunctions and velocity potentials are introduced to better depict the fluxes related to the angular momentum transferred at the ground during an event of mountain or friction torque.

The variance of the total flux divergence per belt is one order of magnitude larger than those of the torques. All variances peak at midlatitudes. As a rule, the structure of the generalized streamfunctions changes little during an event; that is, the structure of the nondivergent part of the fluxes is stable. That of the divergent part, as represented by the velocity potential, undergoes a rapid change near the peak of a torque event. Positive friction torque events in midlatitude belts are preceded by a divergence of angular momentum fluxes in that belt, which is linked to the anticyclonic mass circulation needed to induce the positive torque. The divergence in the belt breaks down shortly before the torque is strongest. Angular momentum is transported upward from the ground after that. Much of the angular momentum generated in a midlatitude belt by positive mountain torques is transported out of the domain, but there is also a short burst of upward transports. Angular momentum anomalies linked to torque events near the equator tend to be symmetric with respect to the equator. Related fluxes affect the midlatitudes of both hemispheres.
\end{abstract}

\section{Introduction}

The zonal mean of the axial angular momentum is a conserved quantity, which can be altered only by transport divergences and/or by fluxes through the earth's surface, that is, by torques. Hence, angular momentum is a good tracer for investigations of atmospheric motions (e.g., Oort 1989). The dynamic interaction of the atmosphere with the earth is described by the angular momentum equations. Hence angular momentum budgets are ideally suited to study the impact of surface friction and mountain torques on the atmosphere. Data have been used to quantify these processes. For example, Peixoto and Oort (1992) present a time mean budget of the zonally averaged axial angular momentum based on observations and relate the torques to the fluxes. It turns out, inter alia, that relative angular momentum is mainly exported from the tropical source

Corresponding author address: Joseph Egger, Meteorologisches Institut, Universität München, Theresienstrasse 37, $80333 \mathrm{Mu}-$ nich, Germany.

E-mail: J.Egger@LRZ.uni-muenchen.de region and returns to the earth in the west wind belts at midlatitudes. Weickmann et al. (2000) present the observed flow and surface pressure patterns lagged with respect to torques. For example, strong mountain torques at midlatitudes are linked to changes of the mass distribution in the Tropics (see also Weickmann 2003). Hence transports of angular momentum must connect the mountain torque region to the tropical "responses." While Oort (1989) and Peixoto and Oort (1992) established such connections for the time mean problem, the investigation of these links in time is still at an early stage. Egger and Hoinka (2004) tackled this time-dependent problem but applied a meridional average to simplify the analysis. Using a statistical version of the angular momentum budget they found downward directed fluxes of negative angular momentum before an event of global positive friction torque. The response to global mountain torques quickly reaches large heights while the ensuing decay proceeds rather slowly. It is the purpose of this article to include the meridional structure of the fluxes and to relate it to local torques.

Theoretical guidance for this endeavor is provided in 
Eliassen (1952) who discusses the atmospheric response to sources of angular momentum specified as local accelerations. In particular, a positive zonal mean source at the ground induces a meridional circulation with equatorward flow at the levels immediately above the source and poleward return flow higher up. Although this is a useful result it is also clear that friction torques cannot be specified as a forcing because they are embedded in and caused by flow structures. Weickmann and Sardeshmukh (1994), Hendon (1995), and, most recently, Feldstein (2001) pointed out that fluctuations in the zonally averaged mean meridional circulation (MMC) are a main generating mechanism for fluctuations of the friction torques. The Ekman equations tell us that equatorward flow must be expected in the boundary layer for positive friction torques, which transports the angular momentum southward. The further route of this angular momentum needs to be clarified. Weickmann et al. (2000) capture the key features of the interaction of MMCs and torques in their global stochastic model by assuming that the friction torque is partly opposed in sign to the global angular momentum deviations. Corresponding modeling of local torque events has not been carried out.

Much theoretical work has been devoted to the analysis of meridional and vertical angular momentum transports toward and away from mountains that exert a torque. However, the main concern so far was with slow, quasi-stationary motions [see Held et al. (2002) for a recent review]. Many mountain torque events are rather short-lived and reflect the passage of Rossby waves over mountains [Lejenäs and Madden (2000); Weickmann (2003); see also Majda et al. (1999); Majda et al. (2003) for stochastic models of this process]. It is an open question how and where the angular momentum thus created is deposited in the atmosphere. Here, we wish to provide some answers to these questions on the basis of data.

The paper is organized as follows. The basic equations are given in section 2. The data are described in section 3. The statistical characteristics of the torques in the various belts are presented in section 4. A detailed analysis of torque events in four selected belts is presented in section 5 .

\section{Equations}

\section{a. Angular momentum budget of annuli}

The equation to be analyzed is the conservation equation for angular momentum in zonally averaged form,

$$
\frac{\partial}{\partial t} \overline{\rho m}+\frac{1}{a} \frac{\partial}{\partial \varphi} \bar{V}+\frac{\partial \bar{W}}{\partial z}=-\sum(\Delta p) a \cos \varphi,
$$

where the bar stands for the zonal integration

$$
\bar{s}=\int_{0}^{2 \pi} s a \cos \varphi d \lambda
$$

of a variable $s$. In (2.1),

$$
m=(u+\Omega a \cos \varphi) a \cos \varphi
$$

( $u$ is zonal velocity; $\Omega=2 \pi$ day $^{-1}$; a is the earth's radius; $\varphi$ is latitude; $m$ is the specific angular momentum; $\rho$ is density);

$$
\begin{aligned}
V & =v \mu, \\
W & =w \mu-a \cos \varphi \tau_{z}+\Omega a^{2} \cos ^{2} \varphi H
\end{aligned}
$$

( $v$ is meridional and $w$ is vertical velocity; $\mu=\rho m$ angular momentum per unit volume) are the meridional and vertical transports; $\tau_{z}$ is the vertical component of the stress; and $H$ is the vertical density transport due to unresolved motions. In principle, (2.4) should also contain transports by unresolved motions, but it is assumed that these are small. The sum on the right-hand side of (2.1) runs over all pressure differences $\Delta p$ across mountain massifs intersected by the line $z=$ a constant and $\varphi=$ a constant for which the zonal integration in (2.1) is performed.

Let us discretize (2.1) by introducing meridional belts and layers in the vertical. We attach the index $i$ to the latitude belt

$-\frac{\pi}{2}+(i-1) \Delta \varphi=\varphi_{i} \leq \varphi \leq-\frac{\pi}{2}+i \Delta \varphi=\varphi_{i+1}$,

where $a \Delta \varphi=1000 \mathrm{~km}$ is the belt's width and the index $j$ to the layer

$$
z_{o}+(j-1) \Delta z=z_{j} \leq z \leq z_{o}+j \Delta z=z_{j}+1,
$$

where $z_{o} \sim 0$ represents a bottom height, and $\Delta z=$ $1000 \mathrm{~m}$. We consider $I=20$ belts and $J=28$ layers. The relative importance of the torques with respect to that of the flux divergences decreases with decreasing $\Delta \varphi$. The torques are all important for the global situation and almost irrelevant for $\Delta \varphi \rightarrow 0$. The choice $\Delta \varphi=9^{\circ}$ ensures that the meridional variability of budgets is captured, but, as will be seen, torques carry still some weight in the budgets. It is a disadvantage of (2.6), (2.7) that the mass contained in the belts and layers decreases with increasing latitude and height. A more even distribution could be obtained by introducing a meridional coordinate $\varphi^{*}=\sin \varphi$ and pressure coordinates. This would, however, remove the mass term [see (2.13) and section 3] from the analysis.

An integration of (2.1) over the volume of annulus $(i, j)$ gives

$$
\frac{d}{d t} \tilde{\mu}_{i j}+\tilde{V}_{i+1 j}-\tilde{V}_{i j}+\bar{W}_{i j+1}-\tilde{W}_{i j}=0
$$

above the peak height of the mountains where we introduce the angular momenta

$$
\tilde{\mu}_{i j}=\int_{\varphi_{i}}^{\varphi_{i+1}} \int_{z_{j}}^{z_{j+1}} \mu a \varphi d \varphi d z
$$

and the fluxes 


$$
\begin{gathered}
\tilde{V}_{i j}=\left.\int_{z_{j}}^{z_{j+1}} \overline{v \mu} d z\right|_{\varphi=\varphi_{i}}, \\
\tilde{W}_{i j}=\left.\int_{\varphi_{i}}^{\varphi_{i+1}} \overline{w \mu} a d \varphi\right|_{z=z_{j}} .
\end{gathered}
$$

The total torque

$$
\tilde{T}_{i}=\tilde{T}_{o i}+\tilde{T}_{f i}
$$

in belt $i$ is the sum of the mountain torque $\tilde{T}_{o i}$ and the friction torque $\tilde{T}_{f i}$. The torques enter in (2.8) if the earth's surface is part of the boundary of an annulus. To facilitate the interpretation of the results we introduce the split

$$
\tilde{\mu}_{i j}=\tilde{\mu}_{w i j}+\tilde{\mu}_{m i j}
$$

of the angular momentum of an annulus in a wind term $\tilde{\mu}_{w}$ that represents the contribution by the zonal momentum and a mass term. The vertical sums $\hat{\mu}_{w i}, \hat{\mu}_{m i}$ over all layers will be considered as well. Finally,

$$
M=\sum_{i j} \tilde{\mu}_{i j}
$$

is the total atmospheric angular momentum.

Data are used to analyze the relation of torques and angular momentum. Thus (2.8) must be cast in a form that is suitable for statistical data analysis. Let $C(a, b \mid \tau)$ denote the expectation $E[a(t) b(t+\tau)]$, that is, the covariance function of $a$ and $b$ with lag $\tau$. If (2.8) is multiplied by a torque $\tilde{T}_{l}(t-\tau)$ in belt 1 , time is redefined, and expectations are formed, the resulting basic equation is

$$
\begin{gathered}
\frac{\partial}{\partial \tau} C\left(\tilde{T}_{l}, \tilde{\mu}_{i j} \mid \tau\right)+C\left(\tilde{T}_{l}, \tilde{V}_{i+1 j} \mid \tau\right)-C\left(\tilde{T}_{l}, \tilde{V}_{i j} \mid \tau\right) \\
+C\left(\tilde{T}_{l}, \tilde{W}_{i j+1} \mid \tau\right)-C\left(\tilde{T}_{l}, \tilde{W}_{i j} \mid \tau\right)=0 .
\end{gathered}
$$

The "response" of the angular momentum in the annulus $(i, j)$ to a torque in belt 1 can be studied using this equation, where $\tilde{T}_{l}$ may be replaced by $\tilde{T}_{o l}$ or $\tilde{T}_{f l}$.

\section{b. Streamfunction and velocity potential}

It is customary to analyze two-dimensional flow problems by introducing a generalized streamfunction $\psi$ and velocity potential $\chi$. Any two-dimensional flux $\mathbf{F}$ can be written

$$
\mathbf{F}=\nabla \chi+\mathbf{n} x \nabla \psi
$$

(n is a unit vector perpendicular to the meridional plane). We introduce volume-averaged representations of $\psi$ and $\chi$, which are adapted to the analysis grid and describe via (2.16) the "flux" $\left[C\left(\tilde{T}_{l}, \tilde{V} \mid \tau\right), C\left(\tilde{T}_{l}, \tilde{W} \mid \tau\right)\right]$ so that

$$
\begin{aligned}
C\left(\tilde{T}_{l}, \tilde{V}_{i j} \mid \tau\right) & =\tilde{\psi}_{i j}-\tilde{\psi}_{i j+1}+\tilde{\chi}_{i+1 j}-\tilde{\chi}_{i j}, \\
C\left(\tilde{T}_{l}, \tilde{W}_{i j} \mid \tau\right) & =\tilde{\psi}_{i+1 j}-\tilde{\psi}_{i j}+\tilde{\chi}_{i j+1}-\tilde{\chi}_{i j} .
\end{aligned}
$$

Note that both $\tilde{\psi}$ and $\tilde{\chi}$ contain contributions from mass and wind term fluxes. There is no separation of eddy and mean flow transports.

A corresponding generalized "vorticity" $\eta$ is given by

$$
\begin{aligned}
\tilde{\eta}_{i j}= & C\left(\tilde{T}_{l}, \tilde{W}_{i j} \mid \tau\right)-C\left(\tilde{T}_{l}, \tilde{W}_{i-1 j} \mid \tau\right)-C\left(\tilde{T}_{l}, \tilde{V}_{i j+1} \mid \tau\right) \\
& +C\left(\tilde{T}_{l}, \tilde{V}_{i j} \mid \tau\right) .
\end{aligned}
$$

Since $\tilde{\eta}$ can be evaluated from the data we can calculate $\tilde{\psi}$ for every lag from

$$
\tilde{\eta}_{i j}=\tilde{\psi}_{i+1 j}+\tilde{\psi}_{i-1 j}+\tilde{\psi}_{i j+1}+\tilde{\psi}_{i j-1}-4 \tilde{\psi}_{i j}
$$

with the boundary condition $\tilde{\psi}=0$ at all boundaries except at the ground. The corresponding condition is given below. Of course, (2.19) corresponds with the standard formula $\eta=\nabla^{2} \psi$. The streamfunction represents that part of the transports that does not affect the "tendency" $d / d \tau C\left(\tilde{T}_{l}, \tilde{\mu}_{i j} \mid \tau\right)$ in (2.15). This tendency is available from the observations and is related to the Laplacian of the velocity potential via

$$
\frac{d}{d \tau} C\left(\tilde{T}_{l}, \tilde{\mu}_{i j} \mid \tau\right)=4 \tilde{\chi}_{i j}-\tilde{\chi}_{i+1 j}-\tilde{\chi}_{i-1 j}-\tilde{\chi}_{i j+1}-\tilde{\chi}_{i j-1} .
$$

Hence, $\tilde{\chi}_{i j}$ can be obtained from (2.20) subject to nogradient boundary conditions except at $\mathrm{z}=0$ where we have to separate that part $C\left(\tilde{T}_{l}, \tilde{T}_{i} \mid \tau\right)_{\chi}$ of the torques that affects the tendency of the angular momentum from the part $C\left(\tilde{T}_{l}, \tilde{T}_{i} \mid \tau\right)_{\psi}$ to which the streamfunction responds. The latter has to satisfy

$$
\sum_{i} C\left(\tilde{T}_{l}, \tilde{T}_{i} \mid \tau\right)_{\psi}=0
$$

It is reasonable to assume that there is no impact by the torques on $\tilde{\chi}$ if the sum over all $C\left(\tilde{T}_{l}, \tilde{T}_{i} \mid \tau\right)$ vanishes. Otherwise we define the sum $S^{+}$over all positive values of $C\left(\tilde{T}_{l}, \tilde{T}_{i} \mid \tau\right)$ and a corresponding negative sum $S^{-}$. If, for example, $S^{+}>\left|S^{-}\right|$then $C\left(\tilde{T}_{l}, \tilde{T}_{i} \mid \tau\right)_{\chi}$ has to be positive in some belts. There is no unique way to resolve this issue. Here, we adopt a minimalistic strategy. For $S^{+}>\left|S^{-}\right|$we assume that all negative torques are balanced by positive ones in other belts within the framework of the streamfunction calculations. These negative torques have, therefore, no impact on $\chi$. What is left are the positive contributions:

$$
C\left(\tilde{T}_{l}, \tilde{T}_{i} \mid \tau\right)_{\chi}=\frac{1}{2}\left(C\left(\tilde{T}_{l}, \tilde{T}_{i} \mid \tau\right)+\left|C\left(\tilde{T}_{l}, T_{i} \mid \tau\right)\right|\right)\left(1+S^{-} / S^{+}\right)
$$

The sum over all terms on the left-hand side is, of course, $S^{+}+S^{-}$, so that (2.22) describes a minimum contribution to $\tilde{\chi}$. It is only in those belts with positive covariances $C\left(\tilde{T}_{l}, \tilde{T}_{i} \mid \tau\right)$ that a contribution to $\tilde{\chi}$ is made via

$$
\tilde{\chi}_{i 2}-\tilde{\chi}_{i 1}=C\left(\tilde{T}_{l}, \tilde{T}_{i} \mid \tau\right)_{\chi}
$$


while

$$
\psi_{i+11}-\psi_{i 1}=C\left(\tilde{T}_{l}, \tilde{T}_{i} \mid \tau\right)_{\psi}=C\left(\tilde{T}_{l}, \tilde{T}_{i} \mid \tau\right)-C\left(\tilde{T}_{l}, \tilde{T}_{i} \mid \tau\right)_{\chi}
$$

If $\left|S^{-}\right|>S^{+}$, (2.22) has to be adapted correspondingly. A different and extreme choice is to assume

$$
\begin{aligned}
& C\left(\tilde{T}_{l}, \tilde{T}_{i} \mid \tau\right)_{\psi}=0, \\
& C\left(\tilde{T}_{l}, \tilde{T}_{i} \mid \tau\right)_{\chi}=C\left(\tilde{T}_{l}, \tilde{T}_{i} \mid \tau\right)
\end{aligned}
$$

so that all torques affect $\tilde{\chi}$ but not $\tilde{\psi}$.

\section{The data}

The data used are contained in the European Centre for Medium-Range Weather Forecasts (ECMWF) ReAnalysis (ERA) dataset covering the period January 1979-December 1992 generated by the ECMWF reanalysis project (Gibson et al. 1997). Wind and density are available at the 31 levels of the hybrid $\sigma$ system of the ERA scheme. The calculation of the torques at the ground can be performed on the basis of these data. As in Egger and Hoinka (2004) all data are transformed to the equidistant surfaces $\mathrm{z}_{j}$ [see (2.7)] with $\Delta z=1000 \mathrm{~m}$ and $j=28$. The annual and semiannual cycle is suppressed by subtracting the corresponding Fourier modes. The four daily data values are averaged so that we have one dataset per day.

The handling of the lower boundary poses a problem in that many of the lower annuli intersect the ERA topography at various points. We impose the rule that interpolated data at height $z_{j}$ do not contribute to the means (2.2) if $z_{j}$ is smaller than the orographic height at this point. In principle a scheme must be designed that distributes the torques over several layers. It has been decided on the basis of the discussion in Egger and Hoinka (2004) to adopt the simplest scheme where all torques are defined at the surface $z_{1}$.

It is an important advantage of the height coordinates that the mass terms can be evaluated as functions of height. That is not possible in pressure coordinates or in $\sigma$ coordinates. The resolved fluxes $\tilde{W}_{i j}, \tilde{V}_{i j}$ are evaluated for each annulus. Note that there is no information available on the vertical flow $\mathrm{H}$ [see (2.5)] nor on the vertical profile of the stress $\tau_{z}$. There is the additional problem that the data do not exactly satisfy (2.15) and, in particular, do not satisfy the vertical balance

$$
\begin{aligned}
\sum_{j=1}^{J}\left[\frac{d}{d \tau} C\left(\tilde{T}_{l}, \tilde{\mu}_{i j} \mid \tau\right)+C\left(\tilde{T}_{l}, \tilde{V}_{i+1 j} \mid \tau\right)-C\left(\tilde{T}_{l}, \tilde{V}_{i j} \mid \tau\right)\right. \\
\left.-C\left(\tilde{T}_{l}, \tilde{T}_{i} \mid \tau\right)\right]=0
\end{aligned}
$$

of (2.15) per belt. Of course, (3.1) is tantamount to requiring

$$
C\left(\tilde{T}_{l}, \tilde{W}_{i J+1} \mid \tau\right)=0 .
$$

To overcome this problem we adjust the horizontal flux terms $C\left(\tilde{T}_{l}, \tilde{V}_{i j} \mid \tau\right)$ in the sense of Gaussian minimization under the constraint (3.1) [see Egger and Hoinka (2005) for further details; see also Hantel and Hacker (1978) and Peixoto and Oort (1992) for similar approaches to this problem]. The vertical transports are calculated from (2.15) as a residual where then (3.2) is ensured. In other words, the vertical fluxes as observed do not enter our evaluations.

\section{Results: Torques and flux divergences}

In this section we wish to present some statistical characteristics of the torques and the divergences in the various belts.

The autocovariance functions of the torques in a belt are presented in Fig. 1. The variance of the mountain torque (Fig. 1a) exhibits three peaks, a small one in Antarctica and two extended ones at midlatitudes (see also Fig. 3 of Iskenderian and Salstein 1998). There is almost no variance at the equator. The decay with increasing lag is quite rapid. There is essentially no signal after, say, 4 days, just as is the case for the global mountain torque (e.g., Egger and Hoinka 2002). The torques are not isolated in their belts, but the covariance $C\left(\tilde{T}_{o i}\right.$, $\tilde{T}_{o j}(0)$ between two belts tends to rather small values over distances of more than three belts. As an example, we show in Table 1 the covariances for belt $15\left(36^{\circ}-\right.$
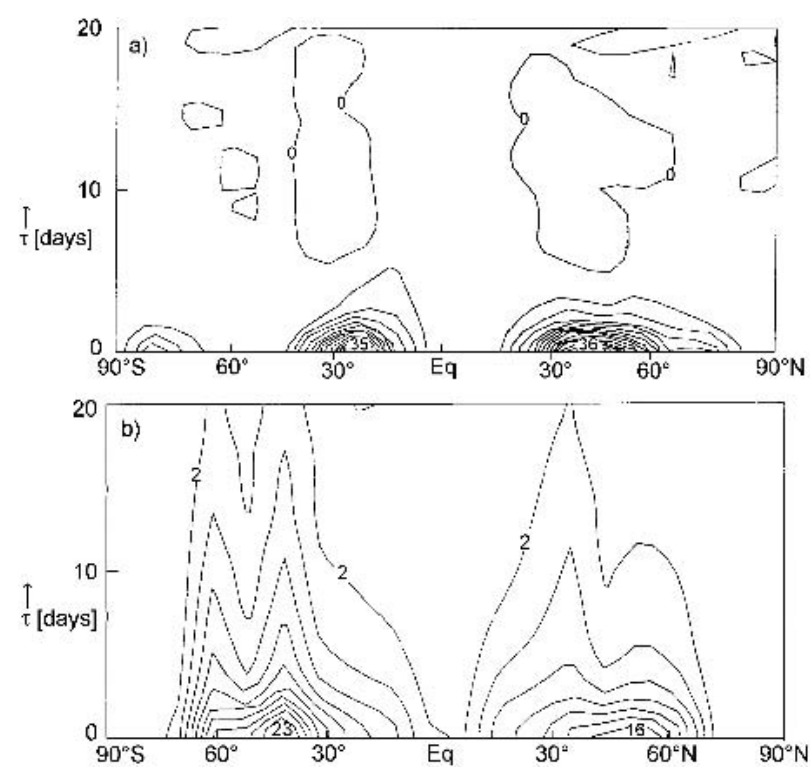

FIG. 1. Autocovariance functions (a) $C\left(\tilde{T}_{o i}, \tilde{T}_{o i} \mid \tau\right)$ of the mountain torques in a belt and (b) $C\left(\tilde{T}_{f i}, \tilde{T}_{f i} \mid \tau\right)$ of the friction torques in $\mathrm{Ha}^{2}\left(1 \mathrm{Ha}=1 \mathrm{Hadley}=10^{18} \mathrm{~kg} \mathrm{~m}^{2} \mathrm{~s}^{-2}\right)$; contour interval $2 \mathrm{Ha}^{2}$; isolines near extrema omitted. The values of the autocovariances are interpolated meridionally as if they were continuous. 
TABLE 1. Covariance of the mountain (friction) torque in belt $15\left(36^{\circ}-45^{\circ} \mathrm{N}\right)$ with mountain (friction) torque in other belts at lag $\tau=0$ in $\mathrm{Ha}^{2}$.

\begin{tabular}{llrrrrrc}
\hline \hline Belt $i$ & 12 & 13 & 14 & 15 & 16 & 17 & 18 \\
$\tilde{T}_{o 15} \tilde{T}_{o i}$ & -0.3 & 4 & 19 & 36 & 15 & 13 & 0.4 \\
$\tilde{T}_{f 15} \tilde{T}_{f i}$ & -2 & -1 & 4 & 15 & 1 & -6 & -2 \\
\hline
\end{tabular}

$\left.45^{\circ} \mathrm{N}\right)$, which vanish almost for belts $12\left(9^{\circ}-18^{\circ} \mathrm{N}\right)$ and $18\left(63^{\circ}-72^{\circ} \mathrm{N}\right)$.

The autocovariances of the friction torques in a belt are displayed in Fig. 1b. Although the variance of friction torques is quite small near the equator the decay with lag is quite slow there (as has been checked). As compared to the mountain torques the decay at midlatitudes is relatively slow but most of the impact is gone after, say, 10 days. It is typical of friction torques that covariances with neighboring belts become negative. An example is provided in Table 1 where $C\left(\tilde{T}_{f 15}\right.$, $\left.\tilde{T}_{f i} \mid 0\right)$ is seen to be negative for $i=17\left(54^{\circ}-63^{\circ} \mathrm{N}\right)$ and $i=13\left(18^{\circ}-27^{\circ} \mathrm{N}\right)$. This feature reflects presumably the impact of the meridional fluxes on the torques.

A figure in analogy to Fig. 1 has been prepared also for the covariances of the vertically integrated flux divergence

$$
\hat{D}_{i}=\sum_{j}\left(\tilde{V}_{i+1 j}-\tilde{V}_{i j}\right),
$$

but the decorrelation times of $\hat{D}_{i}$ are so short that it is better to depict a few autocovariances $C\left(\hat{D}_{i}, \hat{D}_{i} \mid \tau\right)$ in Fig. 2. The variances of these divergences range from $\sim 100 \mathrm{Ha}^{2}$ at the equator (belt $10 ; 9^{\circ} \mathrm{S}-0^{\circ}$ ) to a maximum of $\sim 280 \mathrm{Ha}^{2}$ at midlatitudes. They amount to a few $\mathrm{Ha}^{2}$ in the polar caps. These variances are much larger than those of the torques. The decay is extremely rapid at midlatitudes (belts 5 and 15 ) with a zero crossing for lags of $\sim 1.5$ day. The memory is somewhat longer near the equator (belt 10).

\section{Results: Angular momenta, transports, and torques}

Let us turn now to the "response" of angular momenta to torques and fluxes.

\section{a. General responses}

The relation of the total angular momentum $\hat{\mu}_{i}$ of a belt to the mountain torque $\tilde{T}_{o i}$ in that belt is presented in Fig. 3a. Here and in all following discussions of covariance functions $C(a, b \mid \tau)$ we will assume for the sake of simplicity that the variable $a$ is positive. The angular momentum is typically negative for negative lags to become quickly positive near $\tau=0$ in accord with the reaction of the global value $\mathrm{M}$ to the global mountain torque (e.g., Egger and Hoinka 2004). Unlike the global response, however, this increase toward a positive maximum is relatively weak in the Southern Hemi-

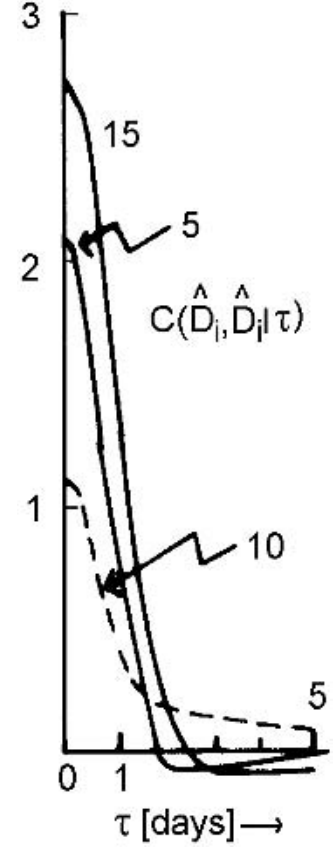

FIG. 2. Autocovariance function $C\left(D_{\dot{v}}, D_{i} \mid \tau\right)$ of the vertically integrated flux divergence $(4.1)$ in $100 \mathrm{Ha}^{2}$ for belts $5\left(54^{\circ}-45^{\circ} \mathrm{S}\right)$, $10\left(9^{\circ} \mathrm{S}-0\right)$, and $15\left(45^{\circ}-54^{\circ} \mathrm{N}\right)$ as a function of lag.

sphere and almost absent in the Northern Hemisphere. Much of the angular momentum generated by the mountain torque in a belt is obviously transported out of this domain.

The substantial negative covariances for $\tau<0$ in Fig. $3 \mathrm{a}$ are not compatible with the hypothesis that the mountain torque can be modeled as a red-noise process with a short decorrelation time $\tau_{c}$ as proposed by Weickmann et al. (2000) for the global situation. They are compatible, however, with the stochastic model of Majda et al. (1999) who derive a negative feedback between mountain torque and $\hat{\mu}_{w}$.

The corresponding covariance pattern $C\left(\tilde{T}_{f i}, \mu \mid \tau\right)$ in Fig. $3 \mathrm{~b}$ shows almost only negative values and is almost strictly symmetric with respect to the line $\tau=0$. As can be seen from Fig. 1b, friction torques act at least for 20 days. Thus the angular momentum decreases in all belts from $\tau=-15$ days, say, till $\tau=0$, despite the input of angular momentum at the ground via the friction torque. That is possible only if angular momentum is removed from the belts by transports and/or by negative mountain torques in that belt for $\tau<0$. For $\tau>0$, fluxes may be less important because $\hat{\mu}_{i}$ increases then in agreement with the positive torque $\tilde{T}_{f i}$. Note that this symmetry with respect to $\tau=0$ is not present for the global friction torque and the global angular momentum $M$. The global covariance $C\left(T_{f}, M \mid \tau\right)$ is positive for $\tau \geq 10$ days and negative for $-20 \leq \tau \leq 10$ days (e.g., Weickmann et al. 2000).

The covariance function $C\left(\hat{D}_{i}, \hat{\mu}_{i} \mid \tau\right)$ as displayed in Fig. $3 \mathrm{c}$ tends to be small for negative lags. It decreases 

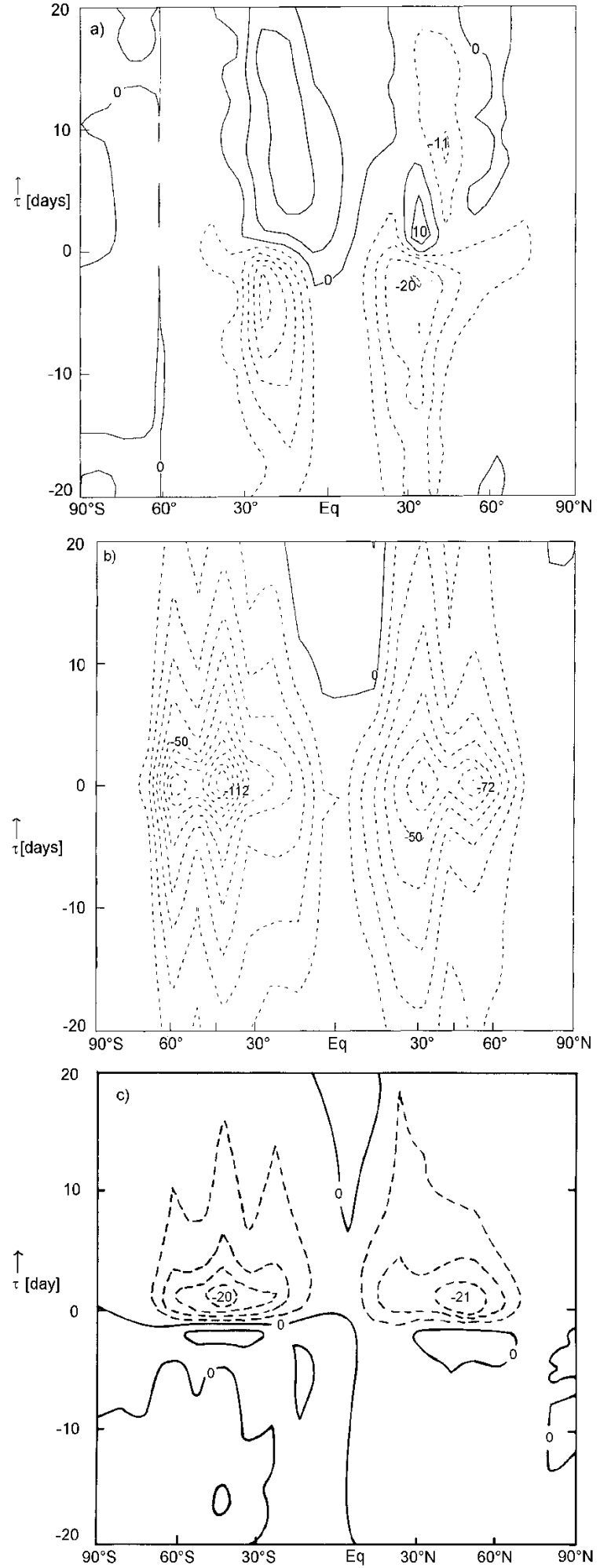

FIG. 3. Covariance functions of torques and divergences in a belt with the vertical sum $\hat{\mu}_{i}$ of the angular momentum in this belt: (a) $C\left(\tilde{T}_{o i}, \hat{\mu}_{i} \mid \tau\right)$, with contour interval $5 \times 10^{5} \mathrm{Ha}^{2} \mathrm{~s}$; (b) $C\left(\tilde{T}_{f i}\right.$, $\left.\hat{\mu}_{i} \mid \tau\right)$, with contour interval $10^{6} \mathrm{Ha}^{2} \mathrm{~s}$; and (c) $C\left(\hat{D}_{i}, \tilde{\mu}_{i} \mid \tau\right)$, with contour interval $5 \times 10^{7} \mathrm{Ha}^{2}$ s. Interpolation as in Fig. 1; negative values dashed. quickly near $\tau=0$ with increasing positive lags to attain its minimum value for $\tau \sim 2$ days. A positive divergence near $\tau=0$ causes a decrease of the angular momentum as should be expected. These minima are about twice as deep as those in Fig. 3b. Reading Fig. $3 \mathrm{c}$ from the top to the bottom, we learn that there is a short period of flux convergence before a moment of positive angular momentum. The ensuing divergence is weak. Altogether Figs. $3 b$ and $3 c$ are in keeping with generally accepted ideas about eddy-induced variations of the relative angular momentum (e.g., Feldstein and Lee 1998; Lorenz and Hartmann 2001; Kim and Lee 2004). The divergences of the vertically integrated angular momentum transport drive the changes of $\hat{\mu}_{w}$. Those anomalies of the wind term induce damping by the surface friction. The time scale of the divergences is short compared to that of the frictional damping.

\section{b. Midlatitude friction torque}

Weickmann et al. (2000) regressed the local wind and mass terms on the global friction and mountain torques. Here, we select torques in individual belts so as to better resolve the local situation. The basic features of an event of friction torque at midlatitudes can be seen from Figs. 4-7. The covariances of the vertically integrated wind and mass terms with the friction torque in belt $15\left(36^{\circ}-45^{\circ} \mathrm{N}\right)$ are displayed in Fig. 4. The covariance $C\left(\tilde{T}_{f 15}, \hat{\mu}_{w} \mid \tau\right)$ decreases with increasing but negative lag in the domain $25^{\circ} \leq \varphi \leq 45^{\circ} \mathrm{N}$ to reach a minimum at $\tau=0$. The situation is similar to that encountered in Fig. 3b. Flux divergences in belt 15 lead to a decrease of $\hat{\mu}_{w}$ there and to an increase of the friction torque. The divergence of the flux in belt 15 is accompanied by convergence in the adjoining stripes to the north and south: In turn, positive anomalies of $\hat{\mu}_{w}$ are created there. At the same time, mass is accumulated to the north of the zone of wind speed reduction and removed in the south (Fig. 4b). Mass and wind variations are approximately in geostrophic balance as has been checked. The changes of the mass term are relatively small when compared to those of the wind term.

The vertical distribution of $C\left(\tilde{T}_{f 15}, \tilde{\mu}_{i j} \mid \tau\right)$ is displayed in Fig. 5 for lag $\tau=0$. As is well known (e.g., Lorenz and Hartmann 2001), the $\mu$ anomalies exhibit an equivalent barotropic structure. The negative covariances in and near belt 15 extend up to the lower stratosphere with little meridional tilt, as do the domains of positive values to the north and south. The extrema are close to the ground except in the southern positive stripe where the maximum is located at a height of $\sim 5$ $\mathrm{km}$. This suggests that the divergence of the transports that causes this pattern is strongest in the lower troposphere. The finding reflects the fact that we are dealing here with angular momentum transports that decrease approximately with $\rho$ with height while many studies of zonal flow variability consider eddy transports of zonal velocity. The pattern in Fig. 5 is fairly stable with respect to variations of the lag. 

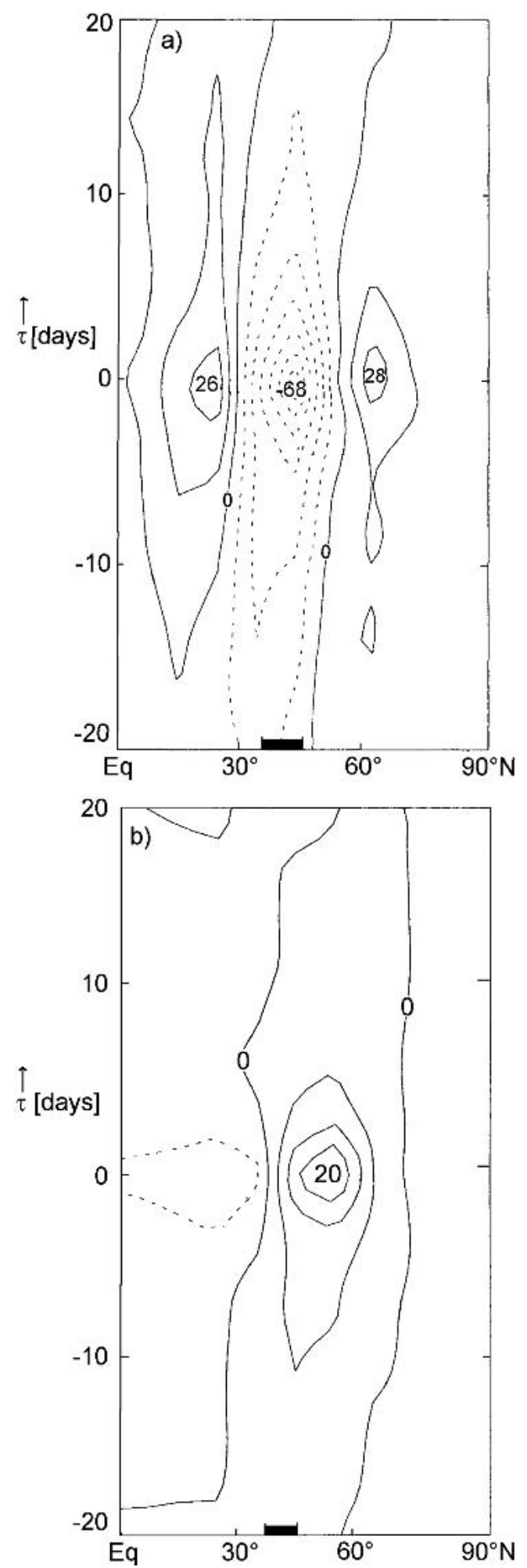

FIG. 4. Covariance functions (a) $C\left(\tilde{T}_{f 15}, \tilde{\mu}_{w i} \mid \tau\right)$ and (b) $C\left(\tilde{T}_{f 15}\right.$, $\left.\tilde{\mu}_{m i} \mid \tau\right)$ of the friction torque in belt $15\left(36^{\circ}-45^{\circ} \mathrm{N}\right)$ with the vertically integrated wind and mass terms in all belts in $10^{5} \mathrm{Ha}^{2} \mathrm{~s}$; lag $\tau$ in days. The bar at the abscissa gives the location of the belt. Northern Hemisphere only; contour interval $10^{6} \mathrm{Ha}^{2} \mathrm{~s}$ in (a) and $5 \times 10^{5} \mathrm{Ha}^{2} \mathrm{~s}$ in (b); negative values dashed.

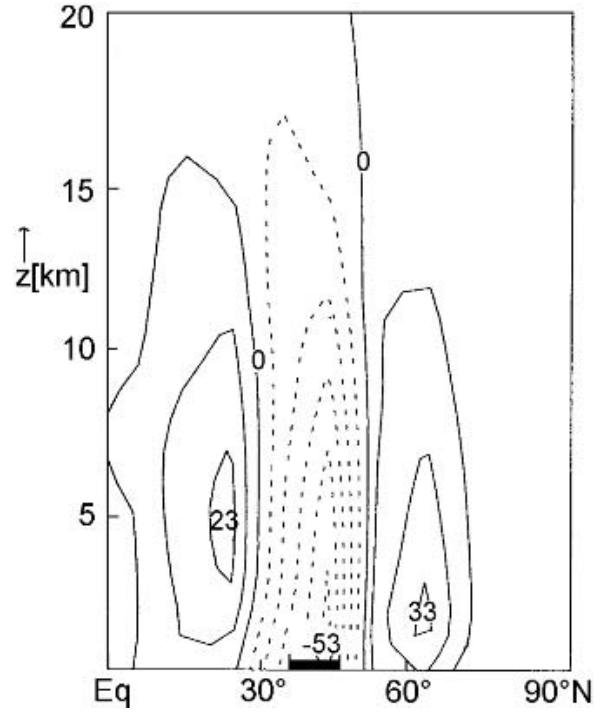

FIG. 5. Covariance function $C\left(\tilde{T}_{f 15}, \tilde{\mu}_{i} \mid 0\right)$ of the friction torque in belt $15\left(36^{\circ}-45^{\circ} \mathrm{N}\right)$ with the angular momentum in $10^{4} \mathrm{Ha}^{2} \mathrm{~s}$ as a function of latitude and height at lag $\tau=0$. Northern Hemisphere only. The bar at the abscissa marks belt 15 . Contour interval $10^{5} \mathrm{Ha}^{2}$ s; negative values dashed.

The covariance $C\left(\tilde{T}_{f 15}, \tilde{\psi} \mid \tau\right)$ exhibits an anticyclonic cell with near-surface northerlies above the belt and a cyclonic one extending across the equator for $\tau<-5$ days (Fig. 6). The "streamlines" intersect the ground in belt 15 such that angular momentum is taken up there. The same amount is deposited mainly to the north without affecting the angular momentum of the atmosphere. It is not possible to separate the contributions of mass term fluxes to $\psi$ from those of wind term fluxes because of the lower boundary condition. Torques act

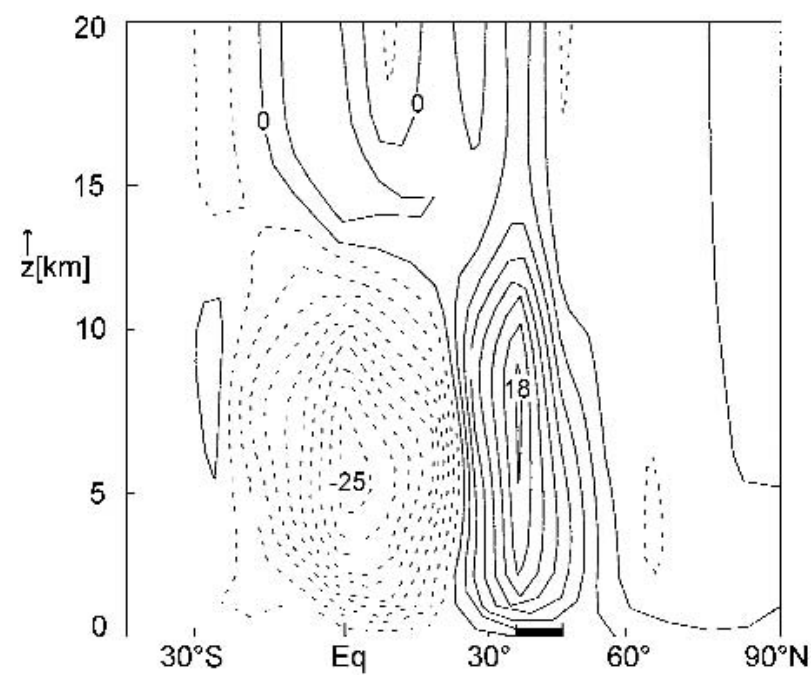

FIG. 6. Generalized streamfunction $\tilde{\psi}$ in $\mathrm{Ha}^{2}$ for lag $\tau=-5$ days and friction torque in belt 15 (bar at abscissa). Contour interval $2.5 \mathrm{Ha}^{2} \mathrm{~s}$; negative values dashed. 


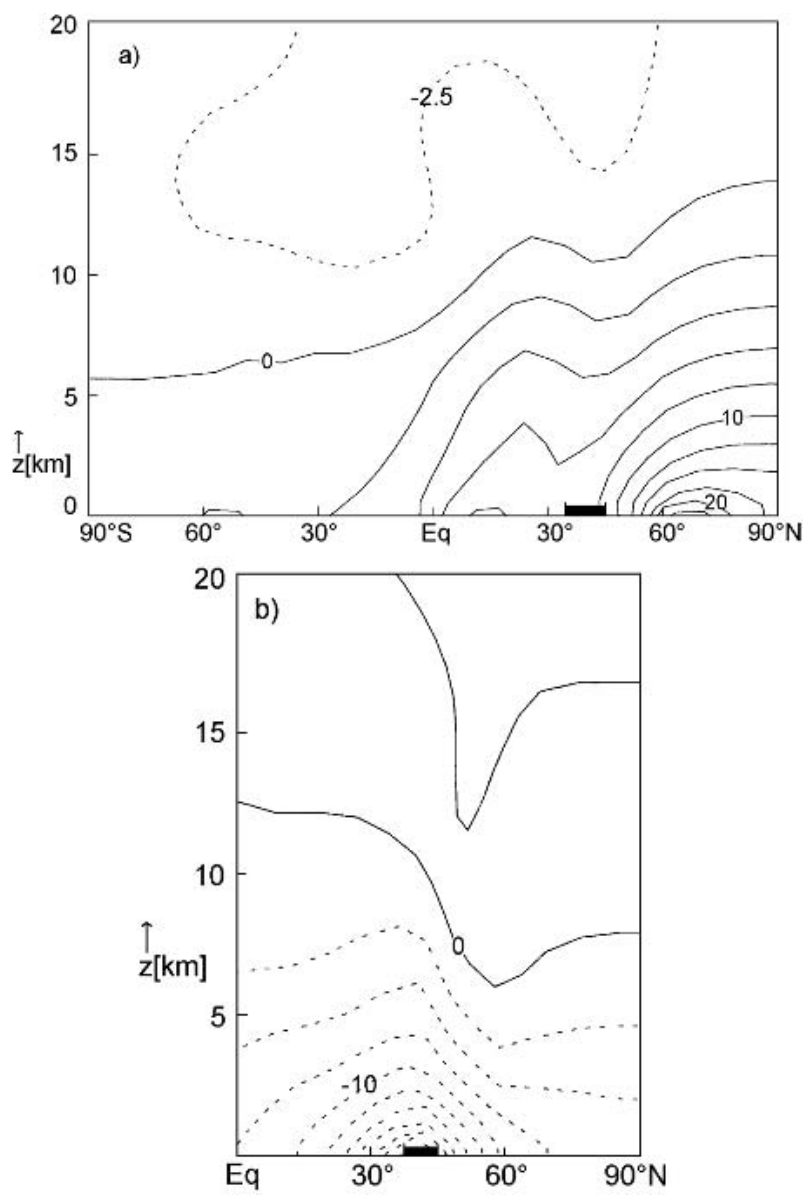

FIG. 7. Generalized velocity potential $\tilde{\chi}$ in $0.1 \mathrm{Ha}^{2}$ for (a) $\tau=$ -5 days and (b) $\tau=0$. Friction torque $\tilde{T}_{f 15}$; contour interval 0.25 $\mathrm{Ha}^{2}$; negative values dashed.

on the total angular momentum. Nevertheless, one can evaluate the contribution of each flux to the vorticity (2.19). It turns out that the mass fluxes dominate clearly. This suggests that there is northerly flow close to the ground in belt 15 as expected and upward (downward) motion to the south (north) of belt 15 . Hence we have here a representation of an MMC similar to that presented by Feldstein (2001). Note, however, that Feldstein calculated the circulation related to global friction torques on the basis of the $\omega$ equation. Here, the circulation is derived directly from the ERA set for the torque in a belt. The structure of the $\psi$ field changes relatively little with increasing lag except that the equatorial cell weakens for $\tau>0$ when compared to the midlatitude cell. The friction torque is negative underneath the "cyclonic" cells in Fig. 6 with negative streamfunctions. Thus part of the positive torque generated in belt 15 is balanced by negative torques in the neighboring stripes of positive angular momentum (see also Fig. $4 a)$.

The velocity potential shows a rather interesting structure at $\tau=-5$ days (Fig. 7a). There are maxima at the ground near $\varphi \sim 25^{\circ} \mathrm{N}$ and $\varphi \sim 75^{\circ} \mathrm{N}$ and a "trough" above belt 15 . This trough represents the decrease of $\mu$ with negative but increasing $\tau$ in this belt (see Figs. 3 and 4) but we see now where the corresponding fluxes are directed. Angular momentum is transported out of belt 15 down toward the ground to the north and south where the $\mu$ anomaly is positive (see Fig. $4 \mathrm{a}$ ) and where the friction torque is negative (not shown). The flux divergence extends throughout the troposphere. This is in keeping with Lorenz and Hartmann (2001) and others who showed that the divergence of eddy meridional zonal wind transports linked to variations of the zonal mean wind extends throughout the troposphere. What is new here is the link to the torques and, of course, the representation of the vertical flux component. Figure 7a supports the findings of Egger and Hoinka (2004) that downward transport of angular momentum precedes an event of positive global friction torque. There is a dramatic change near $\tau=0$ when $\tilde{\chi}$ becomes negative in the troposphere and positive above (Fig. 7b). There is just one minimum of $\tilde{\chi}$ in belt 15 . Angular momentum is transported radially away from the source region in belt 15. The "trough" is still found in the upper troposphere farther north. The dynamic causes for this switch are not known. Note, in particular, that the streamfunction pattern does not undergo such dramatic changes. A consideration of the covariance function $C\left(\tilde{T}_{f i}, D_{i} \mid \tau\right)$ of friction torque and divergence per belt shows that this rapid breakdown of the divergence near $\tau=0$ is common at midlatitudes. The divergence reaches its maximum about 2 days before the positive friction event to become unimportant and mainly negative for $\tau \geq 0$.

The calculations of $\tilde{\psi}$ and $\tilde{\chi}$ have been repeated with the extreme condition (2.25) that excludes any transfer to the ground via the $\psi$ field. This choice has little impact on the streamfunction, which reflects mainly the distribution of the vorticity $\eta$. The velocity potential, however, has to connect the additional sources and sinks at the bottom. This leads in the case of Fig. 7a to an increase of $\tilde{\chi}$ near the equator in the lowest $2 \mathrm{~km}$ and to a corresponding near-surface minimum covering belts 14 and 15 . Figure $7 \mathrm{~b}$ is altered as well, and a maximum of $\tilde{\chi}$ is established in the polar domain.

A corresponding analysis for belt 6 in the Southern Hemisphere reveals that an event of friction torque is embedded in quite similar statistical relations as in the north. In particular, it is mainly Antarctica where the downward angular momentum transports linked to $\tilde{\chi}$ are directed for negative lags in analogy to Fig. 7 a.

\section{c. Midlatitude topography}

Belt $14\left(27^{\circ}-36^{\circ} \mathrm{N}\right)$, with its large variance of the mountain torque (Fig. 1a), is chosen to investigate the statistical characteristics of the atmosphere during an event of mountain torque at midlatitudes. The covariance $C\left(\tilde{T}_{o 14}, \hat{\mu}_{w} \mid \tau\right)$ is shown in Fig. 8a. The mountain 

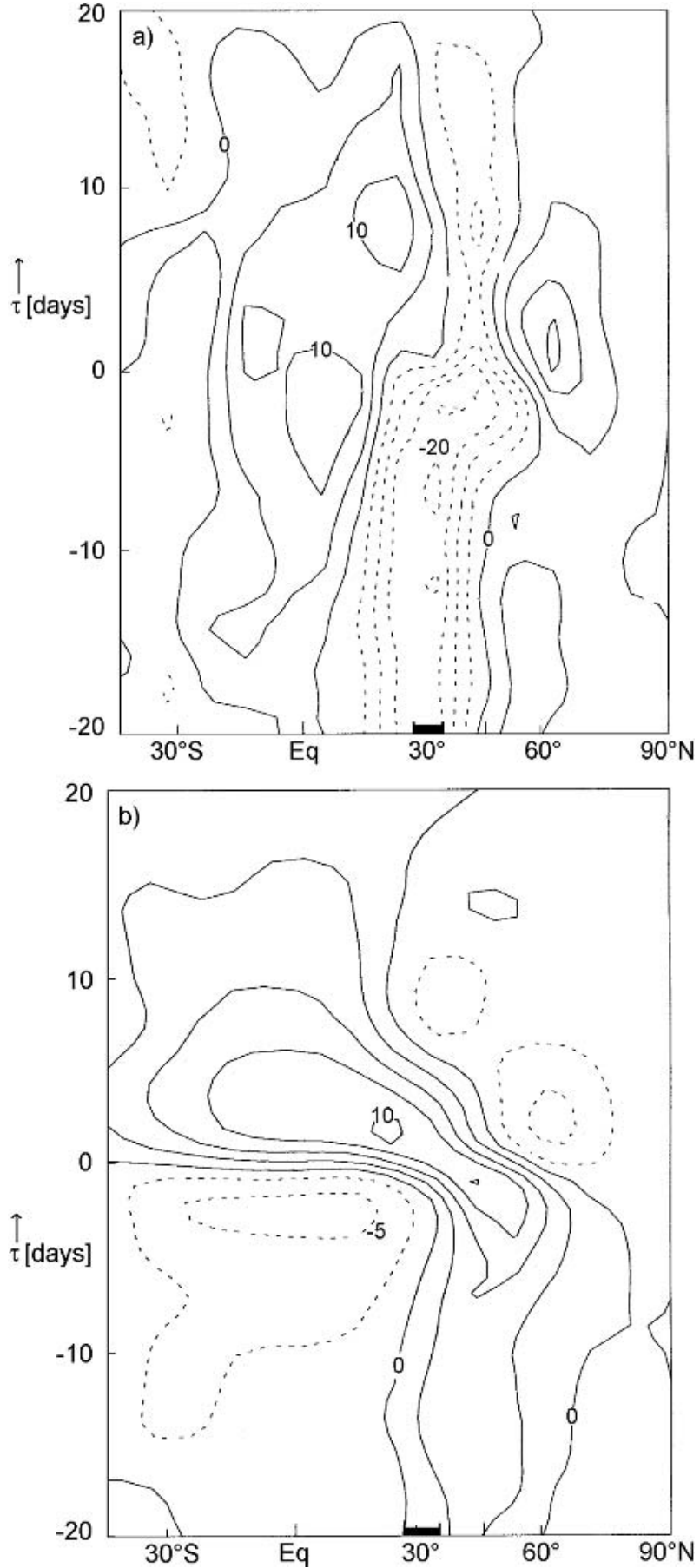

FIG. 8. Covariance function (a) $C\left(\tilde{T}_{o 14}, \hat{\mu}_{w} \mid \tau\right)$ and (b) $C\left(\tilde{T}_{o 14}\right.$, $\left.\hat{\mu}_{m} \mid \tau\right)$ of the mountain torque in belt $14\left(27^{\circ}-36^{\circ} \mathrm{N}\right.$ indicated by bar) and the vertically integrated wind and mass terms in $10^{5}$ $\mathrm{Ha}^{2} \mathrm{~s}$; lag $\tau$ in days. Contour interval $5 \times 10^{5} \mathrm{Ha}^{2} \mathrm{~s}$ in (a) and $2.5 \times 10^{5} \mathrm{Ha}^{2} \mathrm{~s}$ in (b); negative values dashed.

torque event is preceded by an extended stripe of negative wind term anomalies and positive friction torque deviations (not shown). As has been argued above, the extension of this stripe to lags less than -20 days shows that the mountain torque is not independent of $\hat{\mu}_{i}$. Theories of topographic instabilities aim at finding such links (e.g., Jin and Ghil 1990) but have not yet reached the stage of being able to explain the pattern in Fig. 8. As stated above, the stochastic model of Majda et al. (1999) for $\beta$-plane channel flow contains a feedback between mountain torque and angular momentum that may help to explain the structures in Fig. 8a. The wind term increases rapidly near $\tau=0$ in belt 14 and the neighboring domains. Even so, values remain negative in belt 15 for $\tau>0$ (see also Fig. 3a). There are fairly broad domains of positive wind term anomalies both to the north and south of belt 14 that must be generated by the convergence of meridional fluxes. The covariance $C\left(\tilde{T}_{o 14}, \hat{\mu}_{m} \mid \tau\right)$ behaves quite similarly to that in Fig. 4b for $\tau<0$ (Fig. 8b) so that the westerly geostrophic wind near the surface is weaker than normal before the event. However, unlike Fig. 4b, there is a rapid meridional mass shift near $\tau=0$. The low south of belt 14 is replaced by a high extending far into the Southern Hemisphere. The high to the north of the belt becomes a low within a few days. This meridional mass shift is in approximate geostrophic balance with the eastward acceleration due to the mountain torque.

The vertical distribution of wind and mass terms is quasi-barotropic, say, 2 days before the event. The vertical integrals in Fig. 8 are representative of the patterns of $C\left(\tilde{T}_{o 14}, \tilde{\mu}_{w} \mid \tau\right)$ and $C\left(\tilde{T}_{o 14}, \tilde{\mu}_{m} \mid \tau\right)$, say, for $\tau=-2$ days at all levels at least up to a height of $15 \mathrm{~km}$. However, this simple picture is lost quickly when the meridional mass exchange occurs near $\tau=0$ (Fig. 9). Remnants of the "original" column of positive mass covariances in the domain $40^{\circ}-60^{\circ} \mathrm{N}$ (see Fig. $8 \mathrm{~b}$ ) are found in the lower stratosphere, but there is now a large positive blob of mass close to the ground in the domain $25^{\circ} \leq$ $\varphi \leq 45^{\circ} \mathrm{N}$, and a negative one to the north. The vertical

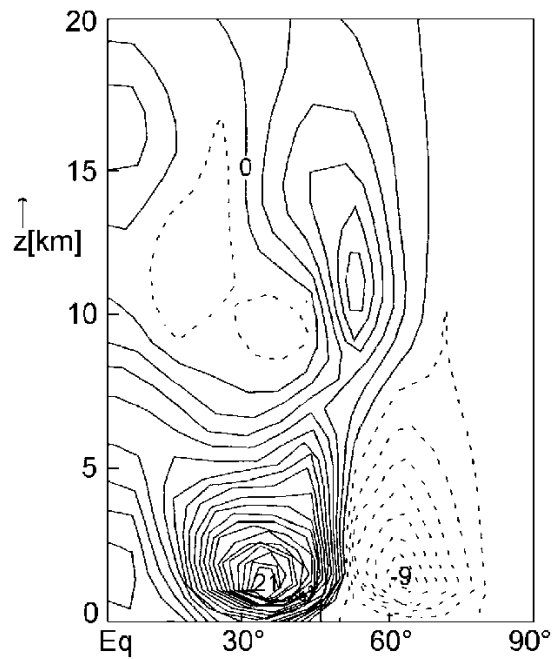

FIG. 9. Covariance function $C\left(\tilde{T}_{o 14}, \tilde{\mu}_{m} \mid \tau\right)$ of mountain torque and mass term per annulus in $10^{5} \mathrm{Ha}^{2} \mathrm{~s}$ as a function of latitude and height for $\tau=0$. Unity contour interval. 
extent of the anomalies is less than the depth of the tropopause. Figure 2 of Weickmann (2003) suggests that the Rossby waves interacting with the Tibetan Plateau, say, experience an anticyclonic rotation so that the high located in the lee during a positive torque event moves toward the southeast. This process is presumably captured by Fig. 9. It explains partly the buildup of positive values of the mass term south of belt 14 in Fig. 8b.

An anticyclonic $\psi$ cell is found in belt 14 throughout the mountain torque event, but a cyclonic cell in the south is evolving as well with increasing lag (not shown). The tendency $d / d \tau C\left(\tilde{T}_{o 14}, \tilde{\mu} \mid \tau\right)$ at $\tau=0$ exhibits a narrow band of large positive values extending from the ground up to a height of $\sim 12 \mathrm{~km}$, which is not seen 2 days earlier nor 2 days later (not shown; see also Egger and Hoinka 2004). The abruptness of the process is also seen in the change of the velocity potential, which has a pronounced minimum at the ground in belt 14 at $\tau=0$. The mountain is a source of angular momentum at that moment. Two days later (Fig. 10) there is a "trough" above the belt, and angular momentum is flowing downward toward a maximum near $70^{\circ} \mathrm{N}$ and also across the equator. This phase of downward propagation is also seen in the global picture of Egger and Hoinka (2004). There exists as yet no model for this process of decay.

\section{d. Tropical friction torque}

Figure 11 shows the covariance $C\left(\tilde{T}_{f 11}, \hat{\mu}_{w} \mid \tau\right)$ of the friction torque in belt $11\left(0^{\circ}-9^{\circ} \mathrm{N}\right)$ with the vertically integrated wind term. As shown above (Figs. 1 and 3 ) the variability of the torques in the equatorial belts is small, but the memory is rather long. The minimum value in Fig. 11 is about one-tenth of that in Fig. 4a. The lateral scale of the wind term anomalies in Fig. 11 is clearly larger than in Fig. 4. The winds in the tropical domain become more easterly throughout the Tropics before the positive friction torque event to gain west-

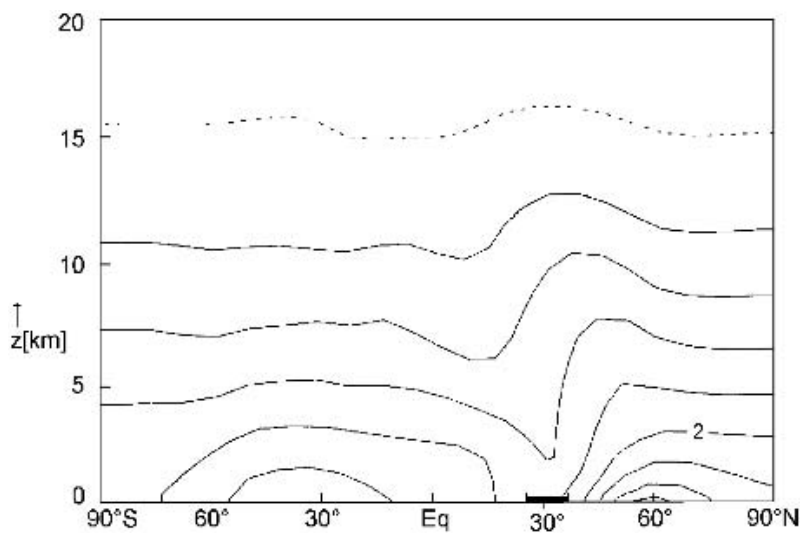

FIG. 10. Covariance function $C\left(\tilde{T}_{o 14}, \tilde{\chi} \mid 2\right)$ in $\mathrm{Ha}^{2}$. Contour interval $0.5 \mathrm{Ha}^{2}$.

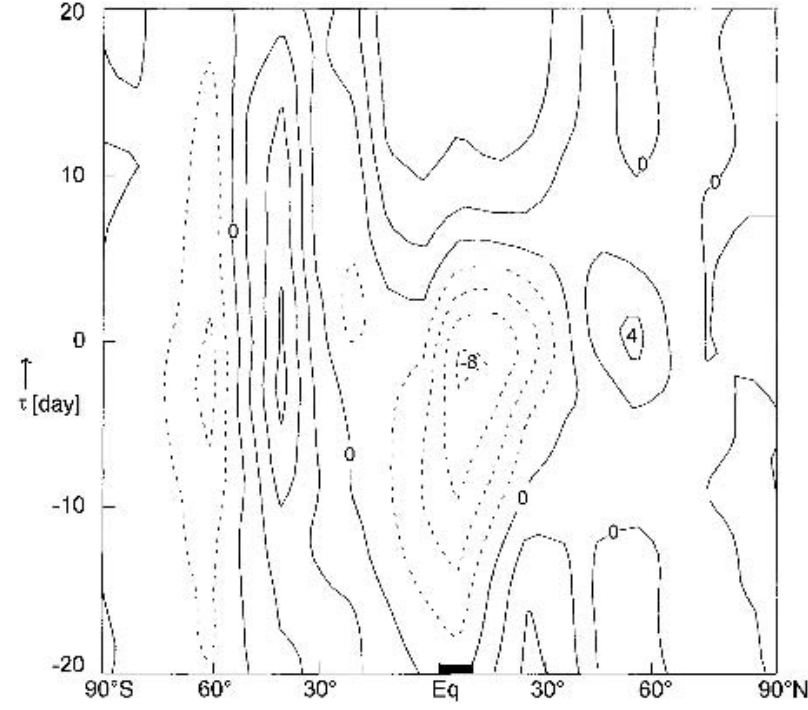

FIG. 11. Covariance function $C\left(\tilde{T}_{f 11}, \hat{\mu}_{w} \mid \tau\right)$ of the friction torque in belt $11\left(0^{\circ}-9^{\circ} \mathrm{N}\right)$ with the vertically integrated wind term in $10^{5}$ $\mathrm{Ha}^{2} \mathrm{~s}$; lag $\tau$ in days. Contour interval $2 \times 10^{5} \mathrm{Ha}^{2} \mathrm{~s}$.

erly momentum for positive lags. There is a narrow stripe of positive anomalies in the Southern Hemisphere and a wider domain in the north. The mass term (not shown) is "geostrophic" in the sense that mass is accumulated to the north of the minimum of the wind term in belt 11 near $\tau=0$. The enlargement of the scales is also clearly visible in the vertical profiles. The covariance $C\left(\tilde{T}_{f 15}, \tilde{\mu} \mid \tau\right)$ is negative throughout the whole of the tropical troposphere for $\tau \leq-3$ days, say. At $\tau=5$ days, the domain of negative anomalies extends to the midlatitudes with narrow positive covariances to the north and south (Fig. 12). Positive anomalies evolve in the upper troposphere, which become dominant for $\tau=10$ days. A certain symmetry with respect to the equator is visible in Figs. 11 and 12. Al-

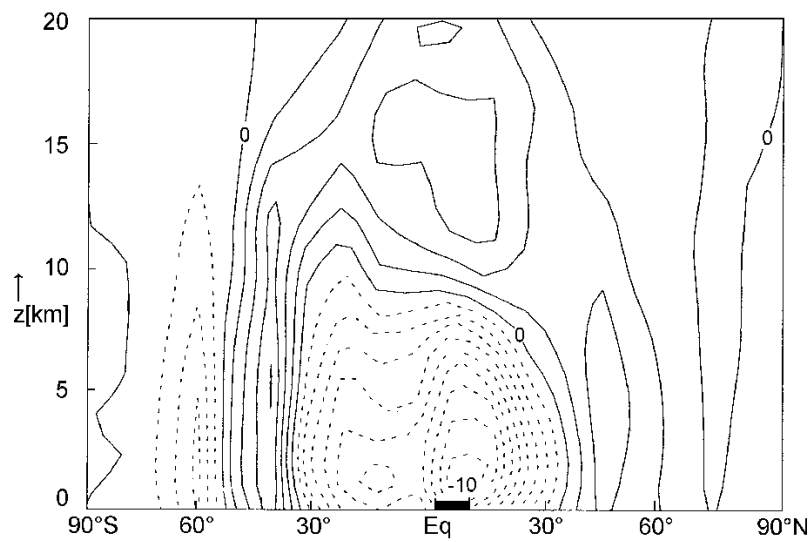

FIG. 12. Covariance function $C\left(\tilde{T}_{f 11}, \tilde{\mu} \mid 5\right)$ of the friction torque in belt 11 and the angular momentum $\tilde{\mu}$ per annulus at lag $\tau=5$ days in $10^{4} \mathrm{Ha}^{2}$ s. Contour interval $10^{4} \mathrm{Ha}^{2} \mathrm{~s}$. 
though the negative anomalies in belt 11 are dominant, a similar but weaker anomaly is seen to evolve in belt 10. A similar situation with reversed signs is found for friction torques in belt 10 . All this suggests that these torques are mainly due to heat sources. Feldstein (1999) demonstrated that convective events at the equator induce indeed friction torques with this quasisymmetric structure with respect to the equator. The basic pattern in Fig. 12 fits this idea quite well. Moreover, linear and also nonlinear models of the atmospheric response to prescribed heat sources near the equator predict dominant westerly inflow close to the ground and easterly outflow near the tropopause (e.g., Gill and Philips 1986). Thus, tropical convection at such scales goes with negative friction torques, and, therefore, Fig. 12 supports these theories.

The streamfunction related to these events is dominated by one anticyclonic cell centered at belt 11 that extends throughout the tropical troposphere. A weak cyclonic cell is seen at $\tau=0$ on the other side of the equator. It is not clear if this pattern reflects a heat source. The related velocity potential (Fig. 13) at $\tau=$ -9 days exhibits two maxima, one near $40^{\circ} \mathrm{S}$ and another one near $40^{\circ} \mathrm{N}$, both in areas where the mountain torque is negative at that lag. There is a minimum in the upper equatorial troposphere so that there is clearly outflow of angular momentum from the tropical belt at that time, which leads to the decrease of the equatorial wind term there for $\tau<0$ (Fig. 11). The velocity potential pattern changes near $\tau=0$ when a minimum at belt 11 begins to dominate. It becomes almost the reverse of Fig. 13 for $\tau=9$ days so that angular momentum moves from the midlatitudes toward the upper troposphere.

Friction torques in belt $10\left(9^{\circ} \mathrm{S}-0^{\circ}\right)$ go also with a quasi-symmetric structure with respect to the equator, but many details differ considerably from the case of belt 11 . An extended discussion is not possible here.

\section{e. Tropical mountain torque}

The variance of the mountain torque in the equatorial belts 10 and 11 is quite small, so that belt $9\left(18^{\circ}-\right.$

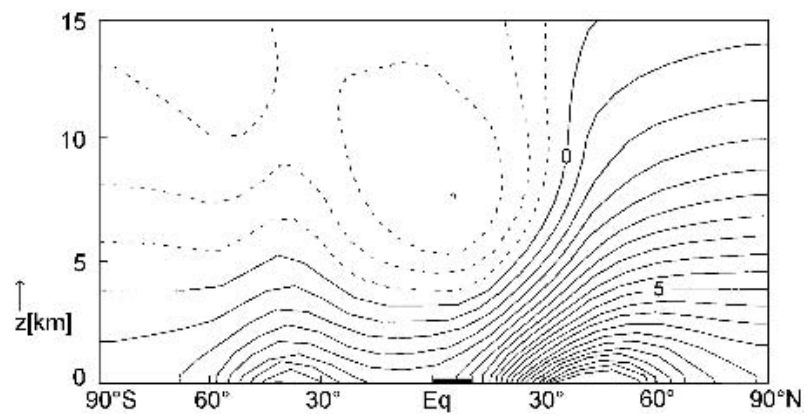

FIG. 13. Generalized velocity potential $\tilde{\chi}$ in $0.1 \mathrm{Ha}^{2}$ for friction torque in belt 11 at lag $\tau=-9$ days. Contour interval $0.05 \mathrm{Ha}^{2}$. $\left.9^{\circ} \mathrm{S}\right)$ is selected to discuss tropical mountain effects. The covariance $C\left(\tilde{T}_{o 9}, \hat{\mu}_{w} \mid \tau\right)$ shows a surprising, almost symmetric structure with respect to the equator (Fig. 14). For $\tau<0$, the vertically integrated wind term decreases near belt 9 but also near belt 12. The positive response to the mountain torques is centered almost at the equator, and not in belt 9 . There are stripes of positive response near $50^{\circ} \mathrm{S}$ and $50^{\circ} \mathrm{N}$. The related vertical structure $C\left(\tilde{T}_{o 9}, \hat{\mu} \mid \tau\right)$ is displayed in Fig. 15 for $\tau=5$ days. The positive anomaly of the angular momentum is almost exactly symmetric with respect to the equator, but there is a negative anomaly near the ground joining the negative branches to the south and north. This negative anomaly is due to the mass term (not shown). Note also that the narrow domains of positive anomalies at midlatitudes tilt toward the equator with increasing height.

The streamfunction is antisymmetric with respect to the equator, with negative values in the south and strong upward transports at the equator (not shown). The velocity potential is negative in the troposphere with a minimum at the ground in belt 11 near $\tau=0$, but the positive contributions $C\left(\tilde{T}_{o 9}, \tilde{T}_{o i} \mid \tau\right)_{\chi}>0$ cover the latitudes $45^{\circ} \mathrm{S} \leq \varphi \leq 30^{\circ} \mathrm{N}$ so that the mountain torques act jointly in a fairly wide domain. At $\tau=10$ days, the $\chi$ pattern is reversed in the decay phase with a minimum in the equatorial stratosphere and various maxima at the ground.

\section{Conclusions}

It has to be stressed before drawing conclusions that polar domains have been excluded as requiring separate study. Moreover, only four situations have been considered in detail.

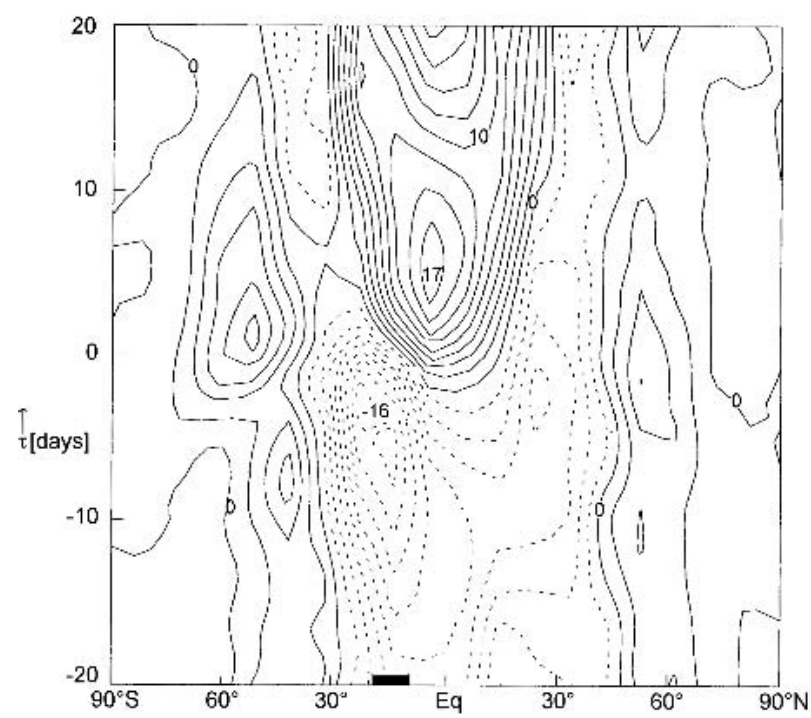

FIG. 14. Covariance function $C\left(\tilde{T}_{o 9}, \hat{\mu}_{w} \mid \tau\right)$ of the mountain torque in belt $9\left(18^{\circ}-9^{\circ} S\right)$ with the vertically integrated wind term in $10^{5} \mathrm{Ha}^{2} \mathrm{~s}$; lag $\tau$ in days. Contour interval $2 \times 10^{5} \mathrm{Ha}^{2}$. 


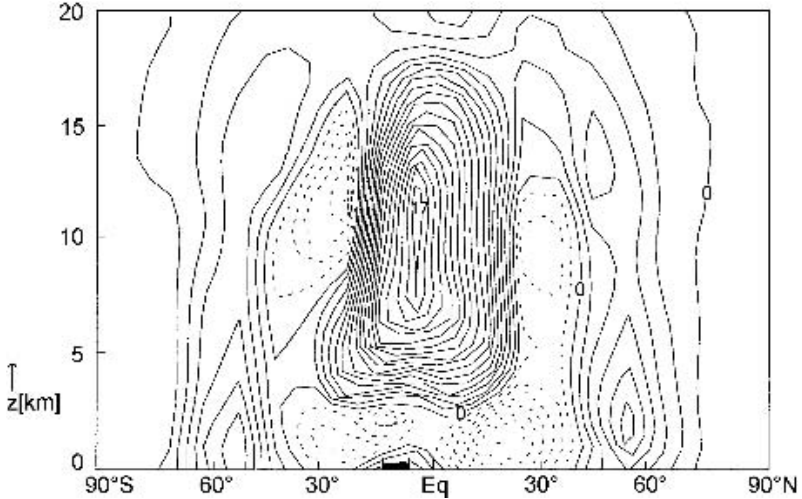

FIG. 15. Covariance function $C\left(\tilde{T}_{o 9}, \tilde{\mu} \mid 5\right)$ of the mountain torque in belt 9 and the angular momentum at $\tau=5$ days in $10^{4}$ $\mathrm{Ha}^{2} \mathrm{~s}$.

1) The variance of the mountain torque in a belt exceeds $10 \mathrm{Ha}^{2}$ in the domains $35^{\circ} \leq \varphi \leq 9^{\circ} \mathrm{S}, 18^{\circ} \leq$ $\varphi \leq 45^{\circ} \mathrm{N}$. Covariances between mountain torques in different belts vanish over distances of more than two belts. The autocovariance of the mountain torque decays within a few days.

2) The variance of the friction torques exceeds $10 \mathrm{Ha}^{2}$ everywhere outside the polar caps and outside the equatorial belts. Strong, mainly negative covariances over large distances are common, particularly so in the Northern Hemisphere. The memory of the friction torques is longer than that of the mountain torques.

3) The variance of the flux divergence $\hat{D}_{i}$ is much larger than those of the torques in the respective belts. The decorrelation time is extremely short.

4) It is a robust feature of all the cases studied that the flux anomalies related to torque events in a belt extend over distances of $10^{4} \mathrm{~km}$ and more.

5) The streamfunction patterns are more persistent than those of the velocity potential, which tend to undergo large changes near $\tau=0$.

6) Positive friction torques at midlatitudes tend to damp negative anomalies of the angular momentum induced by meridional divergences of the angular momentum flux. In particular, $C\left(T_{f i}, \hat{\mu}_{i} \mid \tau\right)$ is almost symmetric with respect to $\tau=0$, with a minimum there in clear deviation from the global situation.

7) Equatorial friction torque events exhibit a pronounced symmetry with respect to the equator, which indicates that they are linked to heat sources. Poleward fluxes and mountain torques are important in removing the angular momentum generated by friction for negative lags.

8) Midlatitude mountains induce an equatorward southward mass shift in the lower troposphere during positive torque events in a belt. The event is preceded by a long phase of negative angular momentum anomalies in that belt. There is a short out- burst of radial angular momentum fluxes away from the mountain near $\tau=0$, which leads to a rapid increase of the angular momentum, particularly so in the lower troposphere. Angular momentum is removed over wide latitude bands in the decay phase.

9) The mountain torque events in the tropical belt 9 $\left(18^{\circ}-9^{\circ} \mathrm{S}\right)$ exhibit a large degree of symmetry with respect to the equator. In particular the area of main angular momentum gains due to the positive mountain torques is centered at the equator and is found above a shallow domain of angular momentum losses linked to mass divergences. There exists little theoretical guidance to explain these phenomena.

Statistical significance of our results has not been established. We rely on Weickmann et al. (2000) who demonstrated for a dataset of similar quality as used here that prominent features tend to be highly significant.

Acknowledgments. The authors are grateful to Klaus Weickmann and an anonymous referee for their perceptive and helpful comments.

\section{REFERENCES}

Egger, J., and K.-P. Hoinka, 2002: Covariance analysis of the global atmospheric axial angular momentum budget. Mon. Wea. Rev., 130, 1063-1070.

- and - 2004: Axial angular momentum: Vertical fluxes and response to torques. Mon. Wea. Rev., 132, 1294-1305.

- and - 2005: The annual cycle of the axial angular momentum of the atmosphere. J. Climate, 18, 757-771.

Eliassen, A., 1952: Slow thermally or frictionally controlled meridional circulation in a circular vortex. Astrophys. Norv., 5, $19-60$.

Feldstein, S. B., 1999: The atmospheric dynamics of intraseasonal length-of-day fluctuations during the austral winter. J. Atmos. Sci., 56, 3043-3055.

_ 2001: Friction torque dynamics associated with intraseasonal length-of-day variability. J. Atmos. Sci., 58, 2942-2953.

—, and S. Lee, 1998: Is the atmosphere driven by an eddy feedback? J. Atmos. Sci., 55, 3077-3086.

Gibson, R., P. Kållberg, S. Uppala, A. Hernandez, A. Nomura, and E. Serrano, 1997: ERA description. ECMWF Reanalysis Project Report Series 1, 86 pp. [Available from ECMWF, Shinfield Park, Reading RGZ 9AX, United Kingdom.]

Gill, A., and P. Philips, 1986: Nonlinear effects on heat-induced circulation of the tropical atmosphere. Quart. J. Roy. Meteor. Soc., 112, 69-91.

Hantel, M., and J. Hacker, 1978: On the vertical transports in the Northern Hemisphere. 2. Vertical eddy transport for summer and winter. J. Geophys. Res., 83 (C3), 1305-1318.

Held, I., M. Ting, and H. Wang, 2002: Northern winter stationary waves: Theory and modeling. J. Climate, 15, 2115-2162.

Hendon, H. H., 1995: Length of day changes associated with the Madden-Julian oscillation. J. Atmos. Sci., 52, 2373-2383.

Iskenderian, H., and D. Salstein, 1998: Regional sources of mountain torque variability and high-frequency fluctuations in atmospheric angular momentum. Mon. Wea. Rev., 126, 16811694.

Jin, F.-F., and M. Ghil, 1990: Intraseasonal oscillations in the extratropics: Hopf bifurcation and topographic instabilities. $J$. Atmos. Sci., 47, 3007-3022.

Kim, H.-K., and S. Lee, 2004: The wave-zonal mean flow inter- 
action in the Southern Hemisphere. J. Atmos. Sci., 61, 10551067.

Lejenäs, H., and R. Madden, 2000: Mountain torques caused by normal-mode global Rossby waves and their impact on atmospheric angular momentum. J. Atmos. Sci., 57, 1045-1051.

Lorenz, D., and D. Hartmann, 2001: Eddy-zonal flow feedback in the Southern Hemisphere. J. Atmos. Sci., 58, 3312-3327.

Majda, A., I. Timofeyev, and E. Vanden Eijnden, 1999: Models for stochastic climate prediction. Proc. Natl. Acad. Sci. USA, 96, 14 687-14 691.

,-- , and -2003 : Systematic strategies for stochastic mode reduction in climate. J. Atmos. Sci., 60, 1705-1722.

Oort, A. H., 1989: Angular momentum cycle in the atmosphere- ocean-solid earth system. Bull. Amer. Meteor. Soc., 70,12311242 .

Peixoto, J., and A. H. Oort, 1992: Climate Dynamics. American Institute of Physics, 520 pp.

Weickmann, K., 2003: Mountains, the global frictional torque, and the circulation over the Pacific-North American region. Mon. Wea. Rev., 131, 2608-2622.

- , and P. Sardeshmukh, 1994: The atmospheric angular momentum cycle associated with a Madden-Julian oscillation. $J$. Atmos. Sci., 51, 3194-3208.

— W. Robinson, and C. Penland, 2000: Stochastic and oscillatory forcing of global atmospheric angular momentum. $J$. Geophys. Res., 105, 15 543-15 557. 